\title{
Selected audio recordings
}

Bradley, S. (1993) Tex Avery Cartoons. Music from the Tex Avery Original Soundtracks Composed by Scott Bradley. Milan [Sound recording: CD].

Cage, J. (2000) Williams Mix from OHM: The Early Gurus of Electronic Music 1948-1980. Ellipsis Arts [Sound recording: CD].

Cage, J. and Patchen, K. (2000) The City Wears a Slouch Hat. Organ of Corti [Audio recording: $\mathrm{CD}$ ].

Negativland (1991) U2: Special Edit Radio Mix and A Capella Mix. Negativland [Sound recording: MP3 file]. Available from: <www.negativland.com> [Accessed 19 November 2005].

Pauline, M. and Jupitter-Larsen, G. (n.d.) Survival Research Laboratories. SubRosa [Audio recording: CD].

Rockmore, C. (1987) The Art of the Theremin. Delos [Sound recording: $\mathrm{CD}$.

Schaeffer, P. (1990) Etude aux chemins de fer (1948) from Pierre Schaeffer: l'oeuvre musicale Vols 1-4. INA-GRM [Audio recording: CD].

Scott, R. (1992) The Music of Raymond Scott. Reckless Nights and Turkish Twilights. Columbia [Sound recording: CD].

Semper, J. (n.d.) Kenotaphion. Charrm [Sound recording: CD].

Stalling, C. (1990) The Carl Stalling Project: Music from Warner Bros. Cartoons 1936-1958. Warner Bros. [Sound recording: CD].

Stalling, C. (1995) The Carl Stalling Project Volume 2. Warner Bros. [Sound recording: $\mathrm{CD}$ ].

Stravinsky, I. (1958) Ebony Concerto, The London Symphony Orchestra. Everest [Audio recording: vinyl].

Varèse, E. (2004) Déserts (1954) and Poème électronique (1958) from Varèse: the Complete Works. Royal Concertgebouw Orchestra, ASKO Ensemble, Ricardo Chailly. Decca [Audio recording: CD]. 